\title{
Comparative Study between Cardioplegic Solution (Custodiol) versus Conventional Cardioplegic Solutions in CABG Patients Y.E.Risk ${ }^{1}$, B.M.Abdelgawad, A.M.Elnahas ${ }^{1}$ and M.M.Melad ${ }^{2}$ \\ ${ }^{1}$ Cardiothoracic Surgery Dept., Faculty of Medicine, Benha Univ., Benha, Egypt \\ ${ }^{2}$ Cardiothoracic Surgery Dept., Nasser Institute, Cairo, Egypt \\ E-Mail:marcoel3reef@yahoo.com
}

\begin{abstract}
Custodiol cardioplegia is appealing for insignificantly intrusive heart medical procedure, as a solitary portion gives a significant stretch of myocardial insurance. In spite of far and wide use in Europe, there is little information affirming its viability contrasted and regular (blood or crystalloid) cardioplegia. We pointed in this examination to set up a correlation among old and most recent methods of cardioplegia with respect to preferences and burdens of both. An imminent accomplice study directed in Cardiothoracic Department, Benha University Hospital during the time frame from March 2018 to May 2020 and included 120 patients of cardiovascular medical procedure which requires cardioplegia and cardiopulmonary detour partitioned into two gatherings: bunch An included 60 patients who got custodial while bunch B included 60 patients who got customary cardioplegia.Our result demonstrated that in gathering the mean EF inside 3 and a half year post-employable (58.8 and 57.9 separately) while in gathering B (58.1 and 57.6 individually) with no measurably distinction. Left ventricular end systolic pressing factor in gathering B post-employable $6 \mathrm{~m}$ (2.7) was lower than that of gathering A (3.5) and there was critical factual distinction $(\mathrm{P}$ esteem $=0.006$ post $6 \mathrm{~m})$. While postoperative discharge portion in the two gatherings indicated unimportant measurable distinction. The utilization of Custodiol is helpful, straightforward, protected and more compelling than monotonous intentionally and without hesitation cardioplegia in ensuring the myocardium in complex cardiovascular tasks particularly postoperative inotropic underpins, ICU stay and emergency clinic stay periods.
\end{abstract}

Keywords: Cardioplegic solution; CABG, Custodiol, Conventional cardioplaegic solutions.

\section{Introduction}

The word cardioplegia joins the Greek cardio meaning the heart, and plegia loss of motion. In fact, this implies capturing or halting the heart so surgeries should be possible in a still and bloodless field. Most normally, notwithstanding, the word cardioplegia alludes to the arrangement used to achieve asystole of the heart, or heart loss of motion [1].

Cardioplegic methods are isolated into cold and worm cardioplegia. The most well-known system for achieving asystole is imbuement of cold cardioplegic arrangement into coronary dissemination. This interaction shields the myocardium from harm during the time of ischemia [2].

Custodiol is an intracellular crystalloid cardioplegic arrangement utilized by certain habitats for myocardial insurance in complex cardiovascular medical procedure and for organ safeguarding in transfer a medical procedure. Histidine-tryptophan-ketoglutarate (HTK), Bretschneider's, or Custodiol is alluring for heart specialists since it is regulated as a solitary portion and is professed to offer myocardial insurance for a time of as long as three hours, permitting execution of complex systems without interference [3].

We pointed in this examination to set up a correlation among old and most recent strategies of cardioplegia with respect to favorable circumstances and detriments of both.

\section{Patients and methods}

A prospective cohort study conducted in Cardiothoracic Department, Benha University Hospital during the period from March 2018 to May 2020 and included 120 patients of cardiac surgery which requires cardioplegia and cardiopulmonary bypassdivided into two groups:

- Group A (60 patients): who received custodiol.
- Group B (60 patients): who received traditional cardioplegia.

\subsection{Methods}

All patients subjected to the following: full history, clinical examination and laboratory investigations (CBC,LFT,RFT,INR, plain chest $\mathrm{x}^{-}$ray, ECG, echocardiography).

\subsection{Intraoperative}

Median sternotomy incision.

- Anticoagulation was established, while the aortic purse string sutures were placed before cannulation with an initial bolus 300-400 IU.kgof heparin sodium to get activated clotting time (ACT) higher than $480 \mathrm{sec}$.

- The cardiopulmonary bypass (CPB) was established by an arterial cannula in the ascending aorta or femoral artery. Venous drainage was obtained via bicaval cannulation through the superior and inferior vena cavae or femoral vein.

- In the beginning for myocardial protection iced slush was used on the anterior surface of heart for topical cooling. An isolating pad was placed between the heart and left side of pericardium containing the phrenic nerve and fluid was aspirated from pericardial cavity regularly to safeguard the phrenic nerve.

- Under CPB and aortic cross clamping, cardioplegic arrest was induced. The cardioplegic solution was administered at a pressure of $5 \mathrm{o}-7 \mathrm{ommHg}$ and given over 5-7 $\mathrm{min}$ while aortic root pressure was kept between 40-50 $\mathrm{mm} \mathrm{Hg}$.

- In Group A (Custodiol) systemic temperature was lowered to $28^{\circ}$. The HTK solutions at $4^{\circ} \mathrm{C}$ were given in an antegrade fashion at an initial perfusion pressure of $80-100 \mathrm{mmHg}$. When the myocardium was at 
standstill, the perfusion pressure was maintained at 40 $60 \mathrm{mmHg}$. A single dose of $25 \mathrm{ml} / \mathrm{kg}$ is given over $5-7$ min in all cases. If there is severe aortic regurge one third is given in the aortic root until the myocardium is at standstill and the other two third is given directly in the coronary ostia according to coronary dominance.

- In group B (traditional cardioplegia) the cardioplegia solution was infused through aortic root systemic temperature was reduced to $28-30{ }^{\circ} \mathrm{C}$ and keep hematocrit between 21-25\%. Blood cardioplegia was given with antegrade route. Blood maintenance cardioplegia mixed at 1:4 (Blood- induction cardioplegic solution) was repeated every $20 \mathrm{~min}$. If there is severe aortic regurge one third is given in the aortic root until the myocardium is at standstill and the other two third is given directly in the coronary ostia according to coronary dominance. Cold Blood cardioplegia was infused at $150-180 \mathrm{ml} / \mathrm{min}$ for an initial dose of 1 litre and subsequent doses of $500 \mathrm{ml}$ each total.

- Mechanical mitral valve and aortic valve had been replaced.

- Before weaning from CPB rewarming was doneuntil nasopharyngeal temperature reached $37^{\circ} \mathrm{C}$ and the hematocrit was maintained between $21-25 \%$. Arterial blood gases (ABGs) were monitored and maintained. For optimal myocardial performance optimal filling pressures were achieved. The aorta was decannulated at completion of half dose of protamine ensuring hemodynamic stability. Proper hemostasis, mediastinal and pleural drains insertion were done.

- Assessment including: Coming off bypass quality (arrhythmias), use of inotropic support, use of D.C and total bypass time and cross clamp time.
The study was approved by the Ethics Board of Benha University and an informed written consent was taken from each participant in the study.

\section{Results}

This study included 120 patients who their mean \pm SD of age in group A was $41.35 \pm 8.42$ and group B was 45.62 \pm 9.51 . There is no statistically significant difference between both groups as regard to preoperative $\mathrm{ECHO}$ data.

The mean $\pm \mathrm{SD}$ of total bypass time was higher in group B $(224 \pm 16.62 \mathrm{~min})$ than in group A $(168.41 \pm 13.53 \mathrm{~min})$ and there was significant statistical difference $(\mathrm{P}$ value $<0.001)$. Also, it shows that the mean \pm SD total cross clamp time was in group B $(84.72 \pm 9.51 \mathrm{~min})$ and it was in group A $(78.55 \pm 7.31 \mathrm{~min})$ and there was insignificant statistical difference (P value 0.013) Table (1).

Table (2) shows that $96.7 \%$ of cases in group A and $100 \%$ of group B has been undergoing DC intra operative, majority of cases of group A returned to sinus rhythm $(86.7 \%)$ while cases of group B only $(23.3 \%)$ and there was significant statistical difference $(\mathrm{P}$ value $<0.001)$. However, one case of group B not returned after DC (ventricular fibrillation) and died.

Regarding to intraoperative data, group A has lower CPB time, serum sodium and IABP use than group B. Also, group A has lower postoperative prolonged $(>24 \mathrm{~h})$ ventilation, sternal wound infection, readmission to ICU, hospital stay (days) and 30-day mortality and higher perioperative blood transfusion and re-exploration for bleeding Table (3).

Table (4) shows that in group A mean of EF within 3 and 6 months post-operative (58.8 and 57.9 respectively) while in group B (58.1 and 57.6 respectively) with no statistically difference. Left ventricular end systolic pressure in group B Post-operative $6 \mathrm{~m}$ (2.7) was lower than that op group A (3.5) and there was significant statistical difference ( $P$ value $=0.006$ post $6 \mathrm{~m}$ ). While postoperative ejection fraction in both groups showed insignificant statistical difference.

\subsection{Ethical committee approval}

Table (1) Distribution of the studied groups regarding TBPT and TCCT.

\begin{tabular}{lccc}
\hline & Group A $(\mathbf{n}=\mathbf{6 0})$ & Group B $(\mathbf{n}=\mathbf{6 0})$ & \multirow{2}{*}{ P value } \\
\cline { 2 - 3 } & Mean \pm SD & Mean \pm SD & \\
\hline TBPT (min) & $168.41 \pm 13.53$ & $224 \pm 16.62$ & $<0.001^{*}$ \\
TCCT (min) & $78.55 \pm 7.31$ & $84.72 \pm 9.51$ & 0.013 \\
\hline
\end{tabular}

Table (2) Distribution of the studied groups regarding coming off bypass.

\begin{tabular}{lcccc}
\hline & & Group A (n = 60) & Group B (n= 60) & \multirow{2}{*}{ P value } \\
\cline { 3 - 4 } & & $\mathbf{N}(\%)$ & $\mathbf{N}(\%)$ & \\
\hline \multirow{2}{*}{ Arrhythmia } & AF & $36(60)$ & $8(13.3)$ & \multirow{2}{*}{0.002} \\
\multirow{2}{*}{ DC } & VT & $24(40)$ & $52(86.7)$ & \multirow{2}{*}{0.751} \\
\multirow{4}{*}{ After DC } & Yes & $58(96.7)$ & $60(100)$ & \\
& Nodal & $8(13.3)$ & $44(73.4)$ & \\
& Sinus & $52(86.7)$ & $14(23.3)$ & $<0.001$ \\
& VT & $0(0)$ & $2(3.3)$ & \\
\hline
\end{tabular}


Table (3) Operative and Clinical Outcomes for the studied Patients.

\begin{tabular}{lccc}
\hline Variable & Group A(n = 60) & Group B $(\mathbf{n}=\mathbf{6 0})$ & P value \\
\hline CPB time (min) & $127.2 \pm 62.3$ & $138.5 \pm 67.2$ & $0.014^{*}$ \\
Intraoperative sodium (mmol/L) & & & \\
Before CPB & $140.7 \pm 52.3$ & $141.1 \pm 53.4$ & 0.362 \\
First sodium after cardioplegia & $131.4 \pm 61.7$ & $135.8 \pm 63.5$ & $0.004^{*}$ \\
Final CPB & $134.9 \pm 73.4$ & $136.8 \pm 52.8$ & $0.017^{*}$ \\
postoperative day 1 & $137.6 \pm 52.7$ & $138.6 \pm 53.2$ & 0.072 \\
ICU stay (days) & $6.57 \pm 8.21$ & $6.72 \pm 8.42$ & 0.742 \\
IABP use & $2(3.3)$ & $4(6.6)$ & 0.082 \\
Clinical stroke & $6(10)$ & $4(6.6)$ & 0.071 \\
Prolonged (>24 h) ventilation & $4(6.6)$ & $2(3.3)$ & 0.082 \\
Sternal wound infection & $2(3.3)$ & $6(10)$ & 0.061 \\
Perioperative Transfusion & & & \\
Red blood cells & $30(50)$ & $26(43.3)$ & 0.361 \\
Fresh frozen plasma & $16(26.7)$ & $12(20)$ & 0.281 \\
Platelets & $14(23.3)$ & $16(26.7)$ & 0.526 \\
Cryoprecipitate & $6(10)$ & $6(10)$ & $>0.999$ \\
Whole blood & $28(46.7)$ & $30(50)$ & 0.271 \\
Re-exploration for bleeding & $12(20)$ & $8(13.3)$ & 0.163 \\
Readmission to ICU & $2(3.3)$ & $4(6.6)$ & 0.082 \\
Hospital stay (days) & $13.4 \pm 11.3$ & $14.8 \pm 13.6$ & 0.527 \\
30 day readmission & $8(13.3)$ & $10(16.6)$ & 0.472 \\
30 day mortality & $4(6.6)$ & $6(10)$ & 0.218 \\
\hline
\end{tabular}

CPB: cardiopulmonary bypass time; POP, postoperative; ICU, intensive care unit; IABP, Intraaortic balloon pump.

Table (4) Distribution of the studied groups regarding to postoperative ECHO.

\begin{tabular}{lllll}
\hline & & Group A $(\mathbf{n}=\mathbf{6 0})$ & Group B $(\mathbf{n}=\mathbf{6 0})$ & \multirow{2}{*}{ P value } \\
\cline { 2 - 3 } EF $(\boldsymbol{\%})$ & Mean \pm SD & Mean \pm SD & 0.421 \\
& Post-operative & $59.2 \pm 8.4$ & $58.7 \pm 5.3$ & 0.873 \\
LVS pressure & 3 months & $58.8 \pm 7.6$ & $58.1 \pm 5.8$ & 0.735 \\
& 6 months & $57.9 \pm 7.2$ & $57.6 \pm 5.1$ & $0.024^{*}$ \\
& Post-operative & $3.7 \pm 0.5$ & $3.2 \pm 0.5$ & $0.016^{*}$ \\
\hline
\end{tabular}

$\mathrm{LA}=$ left atrial ; LVS= Left ventricular end systolic.

\section{Discussion}

Histidine-Tryptophan-Ketoglutarate (HTK)(Custodiol) is an answer dependent on the intracellular degree of electrolytes, proposed by Bretschneider during the 1970s. As of late, Custodiol has been utilized as a multi-organ protection arrangement as it builds perfusion to givers' kidney, liver and pancreas just as cardioplegia in cardiovascular medical procedure in a few nations yet not all around all through the world [4].

Our examination demonstrated that conventional cardioplegia bunch was more established in age and no genuinely critical contrast among custodiol and customary cardioplegia bunch as respect to preoperative clinical condition, with high pervasiveness of hypertension, dyslipidemia, myocardial localized necrosis, pneumonic hypertension and NYHA III in custodiol than customary cardioplegia gathering.

Rather than our examination, Pizanoet al [5] detailed that The blood cardioplegia bunch was essentially more established, and comprised of a more noteworthy number of men with a higher pervasiveness of hyperten $\neg$ sion, diabetes, dyslipidemia, history of myocardial localized necrosis, and marginally lower estimations of discharge part. Dynamic endo $\neg$ carditis, congestive cardiovascular breakdown, and higher estimations of creatiᄀnine were more pervasive in the HTK arrangement gathering.

The investigation in our grasp indicated that $96.7 \%$ of cases in custodiol gathering and $100 \%$ of conventional cardioplegia bunch have been going through DC intra usable, larger part of instances of custodiol bunch got back to sinus musicality $(86.7 \%)$ while cases of customary cardioplegia bunch just $(23.3 \%)$ and there was critical factual contrast ( $\mathrm{P}$ esteem $<0.001$ ). Notwithstanding, one instance of conventional cardioplegia bunch not returned after DC (ventricular fibrillation) and kicked the bucket.

Our examination demonstrated that custodiol bunch has lower cardiopulmonary detour time and intraoperative sodium than customary cardioplegia bunch with genuinely huge distinction between both groups. This finding can be clarified by the continuous interferences essential for the tedious organization of conventional cardioplegia as 
opposed to a solitary beginning portion of HTK arrangement.

In certain examinations, hyponatremia has been accounted for follow $\neg$ ing organization of HTK answer for myocardial assurance, and there is some worry about its expected harmful impact, just as the perils of its intense revision [6].

In the current investigation, we found that intense and transient hyponatremia much of the time happened after HTK organization, yet this hyponatremia most likely has insignificant clinical significance as the serum sodium increments to an ordinary level toward the finish of CPB without a particular therapy [7].

The current investigation uncovered that custodiol bunch has lower intraoperative and postoperative confusions as ICU stay, intra-aortic inflatable siphon use, sternal injury disease, readmission to ICU, clinic stay, 30-day readmission and 30-day mortality than customary cardioplegia gathering.

Liu et al [6] likewise found that there was more limited length-of-stays in the emergency unit less mortality with HTK cardioplegia contrasted with without a second thought cardioplegia and there was like our examination. Be that as it may, Kuslu et al. discovered the two gatherings had similar outcomes with respect to span of mechanical ventilation and length of ICU stay and that was unique in relation to our investigation [8].

In our investigation, postoperative confusions, for example, sternal injury contamination up to death were higher in blood cardioplegia gathering. Sansone et al. revealed that lower mortality in patients going through complex cardiovascular medical procedure utilizing Custodiol contrasted and that patients utilizing blood cardioplegia and this was like our investigation [9].

In this investigation, postoperative ECHO information including EF and LV capacity were preferred in HTK bunch over without a second thought cardioplegia bunch demonstrating that solitary portion of HTK arrangement gives preferred myocardial assurance in DVR over monotonous dosages of blood cardioplegia. Fannelop and associates randomized 16 pigs put on cardiopulmonary detour (CPB) to cardioplegic capture with a solitary portion of Custodiol or discontinuous without hesitation cardioplegia. Pigs accepting Custodiol had lower heart files, ventricular capacity and higher troponin-T discharge in the initial four postoperative hours contrasted and pigs getting with a savage cruelty cardioplegia [10].

$\mathrm{Be}$ that as it may, Radovits et al. shown the powerlessness of Custodiol answer for offer a sensible assurance and conservation of endothelial capacity after long haul ischemic capacity. Hoyer et al. affirmed that there was no critical contrasts in postoperative results between both cardioplegic arrangements and there was unique in relation to our examination [11].

\section{Conclusion}

The utilization of Custodiol is helpful, basic, protected and more successful than dull without remorse cardioplegia in securing the myocardium in complex cardiovascular activities particularly postoperative inotropic underpins, ICU stay and clinic stay periods.

\section{References}

[1] M.M.Gebhard, C.J.Preusse, P.A.Schnabel, H.J.Bretschneider. Different effects of cardioplegic solution HTK during single or intermittent administration. The Thoracic and Cardiovascular Surgeon., Vol. 32(05), PP.271-6,1984.

[2] H.Reichenspurner, C.Russ, P.Uberfuhr, G.Nollert, A.chlüter, B.Reichart. Myocardial preservation using HTK solution for heart transplantation. A multicenter study. European Journal of Cardio-thoracic Surgery: Official Journal of the European Association for Cardiothoracic Surgery, Vol.1;7(8), PP.414-9,1993.

[3] H.J.Bretschneider, G.Hübner, D.Knoll, B.Lohr, H.Nordbeck, P.G.Spieckermann. Myocardial resistance and tolerance to ischemia: physiological and biochemical basis. The Journal of cardiovascular surgery, Vol. 1;16(3), PP.241-60,1975.

[4] B.Braathen, A. Jeppsson, H.Scherstén. One single dose of histidine-tryptophan-ketoglutarate solution gives equally good myocardial protection in elective mitral valve surgery as repetitive cold blood cardioplegia: a prospective randomized study. J Thorac Cardiovasc Surg, Vol.141, PP.995-1001,2011.

[5] A.Pizano, F.R.Montes, M.Carreño, D.Echeverri, J.P.Umaña. Histidine-tryptophan-ketoglutarate solution versus blood cardioplegia in cardiac surgery: a propensity-score matched analysis. InHeart Surg Forum, Vol.2, PP. 158-164,2018.

[6] J.Liu, Z.Feng, J.Zhao. The myocardial protection of HTK cardioplegic solution on the long-term ischemic period in pediatric heart surgery. ASAIO J, Vol.54, PP.470-3,2008.

[7] G.Lindner, B.Zapletal, C.Schwarz, W.Wisser, M.Hiesmayr, A.Lassnigg. Acute hyponatremia after cardioplegia by histidine-tryptophane-ketoglutarate-a retrospective study. Journal of cardiothoracic surgery, Vol.7(1), PP.1-5,2012.

[8] S.Kuslu, P.Zeyneloglu, A.Pirat. Comparison of the effects of histidine-triptophan-ketoglutarate solution and crystalloid cardioplegia on myocardial protection during pediatric cardiac surgery. Critical Care, Vol.18, PP.178,2014.

[9] F.Sansone, G.Punta, F.Parisi, G.M.Dato, E.Zingarelli, F.R,loco. Right minithoracotomy versus full sternotomy for the aortic valve replacement: preliminary results. Heart, Lung and Circulation. , Vol.1;21(3), PP.16973,2012 .

[10] T.Fannelop, G.O.Dahle, P.R.Salminen, C.A.Moen, K.Matre, A.Mongstad. Multidose cold oxygenated blood is superior to a single dose of Bretschneider HTKcardiop legia in the pig. The Annals of thoracic surgery, Vol.1;87(4), PP.1205-13,2009.

[11] T.Radovits, L.N.Lin, J.Zotkina, A.Koch, U.Rauen, G.Köhler. Endothelial dysfunction after long-term cold storage in HTK organ preservation solutions: effects of iron chelators and $\mathrm{N}$ - $\alpha$-acetyl-L-histidine. The Journal of heart and lung transplantation. , Vol.27(2), PP.20816,2008 . 